\title{
Remote temperature measurement of highly reflecting objects in outdoor conditions
}

\author{
by K. CHRZANOWSKI
}

Institute of Optoelectronics, 01-489 WARSAW, Poland

\begin{abstract}
A new method for contactless temperature measurement using multispectral infrared pyrometers was presented by V. TANK a few years ago. The method is advantageously applied to highly reflecting objects of moderate temperatures. Good accuracy was achieved but only for indoor conditions. The method assumes that the environment is a blackbody. The assumption cannot be acceptable for some industrial cases, especially for outdoor conditions as the sky radiation does not fulfil Planck law. A new method acceptable also for outdoor conditions has been developed.
\end{abstract}

\section{Introduction}

Radiation from any object consists of two components: the radiation emitted by the object and the radiation reflected by the object [Figure I]. The first component depends on the two parameters of the object: its temperature $T_{o}$ and emissivity $\varepsilon_{o}$. The second component is generally the reflected radiation of environment of the object (the radiation of the Sun, sky, the neighbouring objects and atmosphere) and depends on many environment parameters.

The first component - the emitted radiation - carries useful information about the parameters of the tested object. The second component - the reflected radiation - does not give us any useful information; it carries disturbances. But unfortunately the reflected radiation cannot be separated from the emitted one and both two components come to the input of the infrared pyrometers or thermographs.

The reflected radiation is negligible when the emissivity of the object is near one and its temperature is high. But the reflected radiation is strong and its influence have to be corrected especially for highly reflecting objects of moderate temperatures.

\section{Classical method}

A new method for contactless temperature measurement using multispectral infrared pyrometers was developed by V. TANK from Germany a few years ago [1]. The method is advantageously applied to the considered case of highly reflecting objects of moderate temperatures (emitting no visible radiation), where conventional methods fail. The method assumes that infrared radiation from the surface of a grey, opaque object is determined by three parameters: its temperature $T_{o}$, its emissivity $\varepsilon_{o}$ and the temperature of its environment $T_{e}$. Measurement of the emerging radiation at $n$ different wavelengths delivers the functional values for a set of $n$ equations:

$$
\begin{aligned}
& y_{1}=\varepsilon_{0} L_{\lambda 1}\left(T_{0}\right)+\left(1-\varepsilon_{0}\right) L_{\lambda 1}\left(T_{e}\right) \\
& y_{2}=\varepsilon_{0} L_{\lambda 2}\left(T_{0}\right)+\left(1-\varepsilon_{0}\right) L_{\lambda 2}\left(T_{e}\right) \\
& y_{3}=\varepsilon_{0} L_{\lambda 3}\left(T_{0}\right)+\left(1-\varepsilon_{0}\right) L_{\lambda 3}\left(T_{e}\right) \\
& \cdots \cdots \cdots \cdots \cdots \cdots \cdots \cdots \cdots \cdots \cdots \cdots \cdots \cdots \cdots \cdots \cdots \cdots \cdots \cdots \cdots \cdots \cdots \cdots \\
& y_{n}=\varepsilon_{0} L_{\lambda n}\left(T_{0}\right)+\left(1-\varepsilon_{0}\right) L_{\lambda n}\left(T_{e}\right)
\end{aligned}
$$




\section{http://dx.doi.org/10.21611/qirt.1992.006}

The $y_{1}, y_{2}, y_{3}, \ldots, y_{n}$ are measured the values of the emerging radiation at $\mathrm{n}$ different wavelengths; object temperature $T_{o}$, its emissivity $\varepsilon_{\mathrm{o}}$ and the temperature of its environment $T_{e}$ are unknowns. To determine the three unknowns $T_{o}, \varepsilon_{O}, T_{e}$ there have to be at least three measured values $n$. Measurement at more than three wavelengths allows the application of balancing calculation to increase the accuracy of the results.

The presented above method was tested experimentally. Very good accuracy has been achieved in laboratory, indoor conditions. The temperature of highly reflected objects of moderate temperatures $\left(300 \div 600^{\circ} \mathrm{C}\right.$ ) have been determined usually with less than $1-2 \%$ deviation from thermocouple measurement. However the accuracy have been much worse for outdoor conditions. The temperature of the objects have been sometimes determined with $20 \div 40 \%$ deviation from thermocouple measurement [Figure 2].

The method assumes that both radiation from the object and environment fully fulfil Planck law. The assumption is fulfilled for indoor condition. However, as it is seen the environment radiation for outdoor conditions does not fulfil that law [Figure 3]. It causes the decrease of accuracy of TANK's method in outdoor conditions.

\section{A new method}

Environment radiation for outdoor conditions usually does not fulfil Planck law. This mean that the temperature of moderate and highly reflecting object of moderate temperatures for outdoor conditions should not be determined from the set of equations (1). But that set can be transformed to the new form:

$$
\begin{aligned}
& y_{1}=\varepsilon_{0} L_{\lambda 1}\left(T_{0}\right)+r_{1}\left(\varepsilon_{0}\right) \\
& y_{2}=\varepsilon_{0} L_{\lambda 2}\left(T_{0}\right)+r_{2}\left(\varepsilon_{0}\right) \\
& y_{3}=\varepsilon_{0} L_{\lambda 3}\left(T_{0}\right)+r_{3}\left(\varepsilon_{0}\right) \\
& \cdots \cdots \cdots \cdots \cdots \cdots \cdots \cdots \cdots \\
& y_{n}=\varepsilon_{0} L_{\lambda n}\left(T_{0}\right)+r_{n}\left(\varepsilon_{0}\right)
\end{aligned}
$$

The $y_{1} y_{2}, y_{3}, \ldots, y_{n}$ are the values of the emerging radiation at $n$ different wavelengths; $\varepsilon_{0} L \lambda_{1}\left(T_{0}\right), \ldots, \varepsilon_{0} L \lambda_{n}\left(T_{0}\right)$ are the enhancements due to radiation emitted by the tested object and the $r_{1}\left(\varepsilon_{0}\right), \ldots, r_{n}\left(\varepsilon_{0}\right)$ are the enhancements due to reflected environment radiation.

There are $n+3$ unknowns in the set of equations (2): object temperature $T_{O}$, its emissivity $\varepsilon_{O}$, environment temperature $T_{e}$, the enhancements due to reflected environment radiation $r_{1}\left(\varepsilon_{0}\right), \ldots, r_{n}\left(\varepsilon_{0}\right)$ and only $n$ measured values of the emerging radiation at $\mathrm{n}$ different wavelengths $y_{1}, y_{2}, y_{3}, \ldots, y_{n}$. This mean that the set of equations (2) cannot be simply solved.

The components $r_{1}\left(\varepsilon_{0}\right), \ldots, r_{n}\left(\varepsilon_{0}\right)$ cannot be simply determined because they depend on the object emissivity $\varepsilon_{O}$ which is also an unknown. But we can create a new set of equations:

$$
\begin{aligned}
& r_{2}\left(\varepsilon_{0}\right) / r_{1}\left(\varepsilon_{0}\right)=k_{1} \\
& \cdots \cdots \cdots \cdots \cdots \cdots \cdots \cdots \\
& r_{n}\left(\varepsilon_{0}\right) / r_{1}\left(\varepsilon_{0}\right)=k_{n-1}
\end{aligned}
$$




\section{http://dx.doi.org/10.21611/qirt.1992.006}

The relationships between components $r_{1}\left(\varepsilon_{0}\right), \ldots, r_{n}\left(\varepsilon_{0}\right)$ do not depend on the emissivity $\varepsilon_{O}$ and they can be experimentally determined. When instead of the tested object we put a cold object of very high reflectivity and approximately identical form as the first one we can assume that only reflected radiation come to the input of the pyrometer. For such case the relationships between components $r_{1}\left(\varepsilon_{0}\right), \ldots, r_{n}\left(\varepsilon_{0}\right)$ equal relationships between the measured values of the emerging radiation at $n$ different wavelengths $y_{1}, y_{2}, y_{3}, \ldots, y_{n}$ :

$$
\begin{aligned}
& y_{2}\left(\varepsilon_{0}=0\right) / y_{1}\left(\varepsilon_{0}=0\right)=k_{1} \\
& \cdots \cdots \cdots \cdots \cdots \cdots \cdots \cdots \cdots \cdots \cdots \cdots \cdots \\
& y_{n}\left(\varepsilon_{0}=0\right) / y_{1}\left(\varepsilon_{0}=0\right)=k_{n-1}
\end{aligned}
$$

Using relationships (3) and (4) the number of unknowns in the set of equations (2) reduces to $u=3$ and the new set can be solved:

$$
\begin{aligned}
& y_{1}=\varepsilon_{0} L_{\lambda 1}\left(T_{0}\right)+r_{1}\left(\varepsilon_{0}\right) \\
& y_{2}=\varepsilon_{0} L_{\lambda 2}\left(T_{0}\right)+r_{1}\left(\varepsilon_{0}\right) k_{1} \\
& y_{3}=\varepsilon_{0} L_{\lambda 3}\left(T_{0}\right)+r_{1}\left(\varepsilon_{0}\right) k_{2} \\
& \cdots \cdots \cdots \cdots \cdots \cdots \cdots \cdots \cdots \cdots \cdots \cdots \cdots \cdots \cdots \\
& y_{n}=\varepsilon_{0} L_{\lambda n}\left(T_{0}\right)+r_{1}\left(\varepsilon_{0}\right) k_{n-1}
\end{aligned}
$$

Measurement at more than three wavelengths allows the application of balancing calculation to increase the accuracy of the results.

The new method has been tested and the results can be seen on the [Figure 4].

As it can be seen the new method has proved its advantage over the classical method for outdoor conditions. Its accuracy is a few times higher in such conditions.

\section{REFERENCES}

[1] TANK (V) and DIETL (H).- Multispectral infrared pyrometer for temperature measurement with automatic correction of the influence of emissivity. Infrared Phys., 30, 3,1989, p. 331-342. 


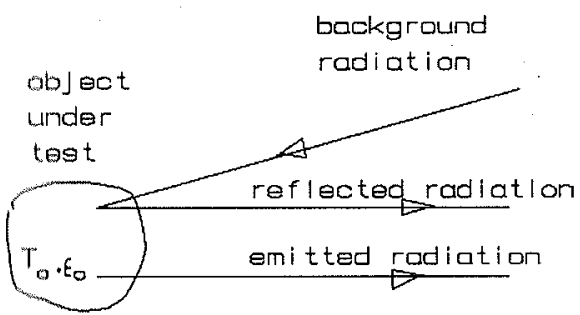

Iig. 1. -Structure of a thermal signal

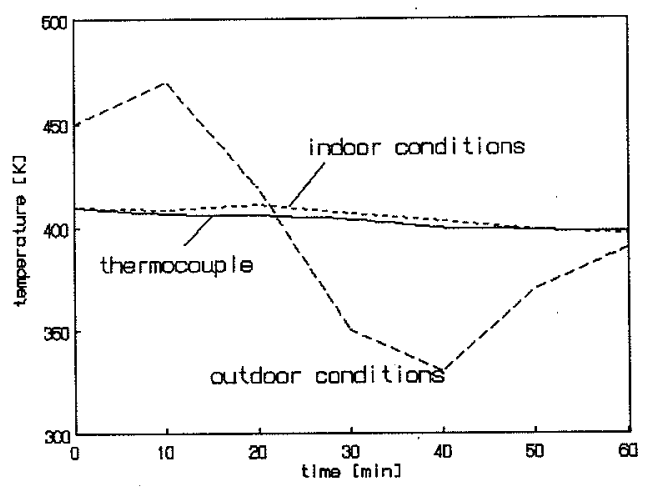

Fig.2. -Temperature of a tested object over time (thermocouple multispectral pyrometer -----)

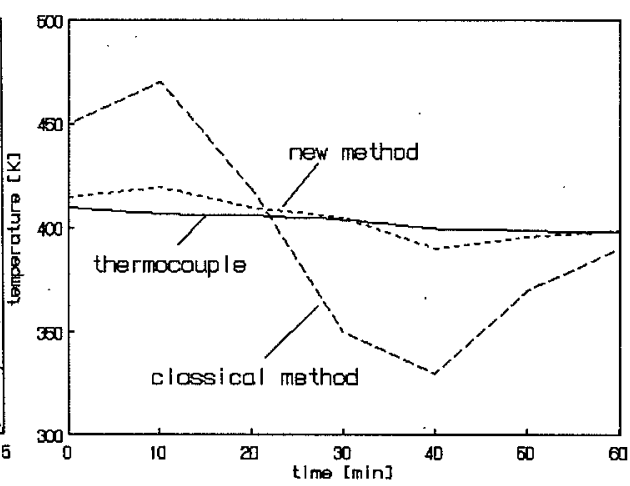

Fig. 4. -Temperature of a tested object measured according to the two being compared methods over time.
$\mathrm{T}_{0}=550 \mathrm{~K}$, emissivity $\varepsilon_{0}=0.1$ ), typical indoor environment (temperature $\mathbf{T}_{\mathbf{e}}=\mathbf{2 9 0} \mathrm{K}$, emissivity $\varepsilon \approx 1$ ) and the sky for a typical autumn day.

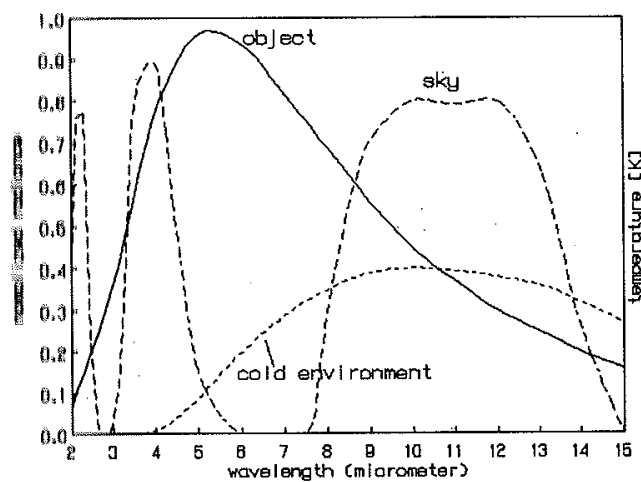

Nig. 3, -Spectral radiance of a highly reflective object (temperature 
http://dx.doi.org/10.21611/qirt.1992.006 